\title{
The Geriatric Emergency Department Intervention model of care: a pragmatic trial
}

\author{
Marianne Wallis ${ }^{1 *}$ (D), Elizabeth Marsden ${ }^{1,2}$, Andrea Taylor ${ }^{1,2}$, Alison Craswell ${ }^{1}$, Marc Broadbent ${ }^{1}$, Adrian Barnett ${ }^{3}$, \\ Kim-Huong Nguyen ${ }^{4}$, Colleen Johnston ${ }^{1}$, Amanda Glenwright ${ }^{5}$ and Julia Crilly ${ }^{6,7}$
}

\begin{abstract}
Background: To evaluate a Geriatric Emergency Department Intervention (GEDI) model of service delivery for adults aged 70 years and older.

Methods: A pragmatic trial of the GEDI model using a pre-post design. GEDI is a nurse-led, physician-championed, Emergency Department (ED) intervention; developed to improve the care of frail older adults in the ED. The nurses had gerontology experience and education and provided targeted geriatric assessment and streamlining of care. The final format included 2.4 full time equivalent nurses working 7 days from $0700 \mathrm{~h}$ to $1730 \mathrm{~h}(1530 \mathrm{~h}$ at weekends). There were three implementations periods: pre-implementation (2012); a developmental phase from January 2013 to August 2015; and full implementation from September 2015 to August 2016. The outcomes measured were disposition (discharged home, admitted or died); ED length of stay; hospital length of stay; all cause in-hospital mortality within 28 days; time to ED re-presentation up to 28 days post-discharge; in-hospital costs. The setting was a tertiary hospital ED, with 385 beds, in Queensland, Australia. Approximately 53,000 patients presented to the ED annually with 20\% aged 70 years and older. All patients over the age 70 who presented to the ED between January 2012 and August $2016(n=44,983)$ were included in the trial.
\end{abstract}

Results: Older persons who presented to the ED when the GEDI team were working had increased likelihoods of discharge (Hazard ratio $(H R)=1.19 ; 95 \% \mathrm{Cl}: 1.13-1.24)$ and reduced $\mathrm{ED}$ length of stay ( $\mathrm{HR}=1.42 ; 95 \% \mathrm{Cl}: 1.33-1.52$ ) compared with those who presented when GEDI were not working. There was no increase in the risk of mortality $(H R=1.01 ; 95 \% \mathrm{Cl}=0.23-4.43)$ or risk of same cause re-presentation to 28 days ( $H R=1.21 ; 95 \%$ Cl: $0.99-1.49)$. The GEDI service resulted in average cost savings per ED presentation of $\$ 35[95 \% \mathrm{Cl}, \$ 21, \$ 49]$ and savings of $\$ 1469$ [95\% Cl, \$1105, \$1834] per hospital admission.

Conclusions: Implementation of a nurse-led physician-championed model of ED care, focused on frail older adults, reduced ED length of stay, hospital admission and if admitted, hospital length of stay and cost, without increasing mortality or same cause re-presentation. These increases were sustained over time and after the initial implementation team had changed roles.

Trial registration: Australian Clinical Trials Registration Number ACTRN12615001157561 - retrospectively registered on 29/10/2015. Data were retrieved via retrospective access to clinical information systems. First data access was on 1/7/2015.

Keywords: Geriatric, Emergency medical services, Nurses' practice patterns, Hospital, Homes for the aged, Delivery of health care, Protocol, Outcomes, Evaluation, Pragmatic paradigm

\footnotetext{
* Correspondence: mwallis@usc.edu.au

${ }^{1}$ School of Nursing, Midwifery and Paramedicine, University of Sunshine

Coast, 90 Sippy Downs Drive, Sippy Downs, QLD 4556, Australia

Full list of author information is available at the end of the article
}

(c) The Author(s). 2018 Open Access This article is distributed under the terms of the Creative Commons Attribution 4.0 International License (http://creativecommons.org/licenses/by/4.0/), which permits unrestricted use, distribution, and reproduction in any medium, provided you give appropriate credit to the original author(s) and the source, provide a link to the Creative Commons license, and indicate if changes were made. The Creative Commons Public Domain Dedication waiver (http://creativecommons.org/publicdomain/zero/1.0/) applies to the data made available in this article, unless otherwise stated. 


\section{Background}

As a consequence of the ageing of the population, older people comprise an increasing proportion of emergency department (ED) presentations [1]. Many frail older persons live in residential aged care facilities (RACFs) but 30 to $50 \%$ live in the community $[2,3]$. When presenting to the ED, frail older adults receive a greater number of tests, spend longer in ED awaiting disposition planning and are at greater risk for medical complications, functional decline and poorer health following discharge $[4,5]$. If RACF residents are hospitalised they suffer higher rates of adverse events and are susceptible to de-conditioning and worsening cognitive state [6-8]. Hospital admission for frail older adults is associated with increased morbidity and mortality $[9,10]$.

Previous studies have indicated ways in which the care of frail older adults experiencing acute illness can be improved $[11,12]$. There are numerous suggestions for improvements in RACFs focusing on enhancing primary care $[1,13-15]$. In the ED there are several studies looking at older persons over the age of 65 , however, these actively exclude RACF residents [16, 17]. The limited previous research on this topic indicates that to improve the care of frail older adults in the ED there should be a single point of contact and structured communication tools [12, 17]; advanced aged care assessment at point of ED entry [11, 12, 17-19]; expert gerontological care by both medical and nursing staff [11, 20, 21]; and streamlining of patient management in $\operatorname{ED}[17,22,23]$. The increased costs of these additional services means that research in this area must also collect data on costs as well as effectiveness.

Clinicians at a regional hospital in Queensland, Australia in collaboration with staff from a local RACF identified that both RACF residents and frail older adults living in the community were experiencing high rates of ED presentation and having worse outcomes than other cohorts. In consultation with university colleagues and the local primary health network (PHN) they designed the Care coordination through Emergency Department, Residential Aged Care and Primary Health Collaboration (CEDRiC) project. This was a two-pronged model of service delivery with interventions in both the RACF and ED. In the ED the Geriatric Emergency Department Intervention (GEDI) was implemented while a Nurse Practitioner Candidate was introduced to the aged care facility.

\section{Methods}

The aim of this study was to evaluate the effectiveness and cost of the GEDI model of service delivery for adults aged 70 years and over, presenting to an ED in regional Queensland, Australia. The protocol for the structure, process and outcome evaluation [24] of the GEDI model has been published [25].

A randomised trial of individual patients was not feasible when considering a change to the model of care for a whole ED, and a cluster-randomised trial would be costly. Hence we undertook a pragmatic trial using a pre-post design that tracked eligible patients before and after the intervention was implemented [26].

\section{Participants and data collection}

De-identified data for all patients aged 70 years and older, who presented to the study ED from January 2012 through to 31st August 2016, were retrieved from Emergency Department Information Service (EDIS; Healthcare Group, $\mathrm{CSC})^{\circ}$ and Hospital Based Corporate Information System (HBCIS; iSoft) databases. Data extraction was undertaken by health service data managers with deterministic linking of the EDIS, HBCIS and financial data. There were three time periods:

1. 1st January to 31st December 2012: prior to development of any aspect of GEDI (hereon referred to as - "pre-intervention or pre-GEDI");

2. 1st January 2013 to 31st August 2015 - the development phase of the intervention during which funding and staffing models changed (hereon referred to as - "interim intervention or interim GEDI"); and

3. 1st September 2015 to 31st August 2016 - (hereon referred to as "full intervention").

To overcome the 'improvement-evaporation effect', whereby the benefits reaped from new practices diminish over time [27] we used data from the immediate period post intervention (interim intervention), when the model was being developed and staffed by the most experienced and dedicated clinicians, but also included the full implementation period which represents a more standard implementation environment.

Independent variables that were used to describe the sample and to build multivariable models to compare outcomes were:

- Demographics - Age, Sex

- Date and time of presentation

- Clinical diagnosis - reason for presentation as ICD10 code - this variable was then mapped to 25 major diagnostic categories

- Arrival by ambulance

- Presented from RACF

- Australasian Triage Score (ATS) $[28,29]$ three groups $1 \& 2,3,4 \& 5$

- Adult Deterioration Detection System (ADDS) Score [28] 
- Time (per 100 days increase across the project)

- Presented to ED during GEDI working hours - Yes/No

- Intervention group (explained below)

\section{Intervention}

The intervention has been described previously $[25,30]$ and is detailed within an Implementation Toolkit [31]. The aims of the model of care are to avoid hospital admission, if appropriate, and where this is not possible, to fast-track admission and medical management. Briefly, the GEDI intervention is delivered in the ED by a multidisciplinary team consisting of an ED physician champion and advanced practice ED nurses who have at least 5 years of experience working with older adults and preferably post-graduate qualifications in gerontology. The GEDI nurses operate as a supplementary sub-speciality team assisting the primary ED nurses and physicians. They target all patients over the age of 70 years especially those transferred from RACFs. They will either receive referrals from the primary care team or will identify patients via the electronic medical records system or via routine rounding. The use of frailty screening was trialled but was not found to be of use for these experienced clinicians.

In consultation, with the ED physician champion they will undertake targeted geriatric assessment (using the aspects of a comprehensive geriatric assessment that are appropriate for the individual patient) and problem formulation. They will then work with the primary ED team to fast-track diagnostic processes and engage the multi-disciplinary team and, where possible, the family in client-centred decision-making. Where necessary GEDI will undertake early referral to specialist care and/ or activate standardised fast track pathways e.g. for orthopaedic surgery or stroke management.

When necessary, GEDI will coordinate admission to a specialty in-patient ward avoiding, where possible, a stay on a medical assessment unit. When admission is not required, the GEDI team may assist the primary care team with hands on care (e.g. wound care, catheter change etc.) and/or liaise with appropriate community or RACF services, to mobilise resources within the patient's home to ensure safe discharge. Finally, the team communicates all ED care and future requirements of care to either the ward or the community care team, including the GP.

The GEDI team also provides an ongoing staff development program for other ED staff. Through a program of in-service education sessions new and junior staff are provided with information about the model of care and are also educated about geriatric syndromes, cognitive assessment and the care pathways used in the department.

\section{Objectives}

The study objectives were to test differences in disposition, ED and hospital length of stay, time to ED re-presentation, all-cause mortality and costs between the groups of patients who presented, to the study ED, in the three time periods.

\section{Outcomes \\ Primary}

- Disposition - discharged home, admitted, died

\section{Secondary}

- ED length of stay- in minutes

- Hospital length of stay - in days

- All cause in-hospital mortality within 30 days of ED presentation

- Time to ED re-presentations up to 28 days

- Cost of hospital admission

\section{Patient and public involvement}

This project and the development of the intervention were a co-design activity that included two members of the public who were over 70 years of age. They are volunteers who spent a lot of their time visiting older adults in aged care facilities and had both personal and proxy experience of the health service and the ED. They not only joined the External Advisory Group set up to guide the development of the intervention and the conduct of the study but also visited the ED and a local aged care facility with members of the research team to discuss issues specific to older adults.

\section{Statistical methods}

An independent statistician, not otherwise involved in the study, provided statistical summaries and analysis. Descriptive statistics were used to describe the participants in the three time periods including frequencies, percentages, appropriate measures of central tendency and distribution.

For the primary outcome, survival analysis was used to jointly model length of stay and disposition, with the three destinations as competing risks [32]. We used survival analysis for ED re-presentations with out-of-hospital mortality as a competing risk. All models adjusted for the patient level factors of gender, age, ATS, season, day of the week and time of presentation. Survival analysis is ideal here because our primary outcomes are times that are subject to censoring from competing risks. Previous analysis have simply categorised time and used logistic regression, for example discharge within $24 \mathrm{~h}$. However, this wastes valuable information and reduces statistical power. We used cumulative incidence curves to look for changes due to the intervention over all times.

Pre-post designs are vulnerable to confounding by other changes over time that may be attributed to the 
intervention [33]. To control for this, we included a linear trend (based on date) in all models to account for gradual changes that are not captured in the individual variables, e.g., experience of healthcare workforce. We also adjusted for season using a sinusoid with an annual cycle to control for the winter peak in morbidity [34]. The survival analyses used Cox proportional hazard survival models. The models' residuals were checked for outliers and correlation over time. We calculated Cook's influential statistic and examined relatively large outliers. We calculated the variance inflation factor and removed variables with a score above five on the basis that they were co-linear. The key outcome was the mean effect of the intervention together with $95 \%$ confidence intervals.

We compared patients who may have received the GEDI intervention to patients for whom it was not available. This "usual care" group could be those who were admitted before the intervention was introduced, or those who were admitted after the intervention but outside the GEDI team's hours. We also expected the intervention to change during the interim and full period, hence we created five categories:

- Pre intervention (control)

- Interim intervention during GEDI working hours (intervention)

- Interim intervention outside GEDI working hours (control)

- Full intervention during GEDI working hours (intervention)

- Full intervention outside GEDI working hours (control)

Hence we had three control groups and two intervention groups. We used the additional control groups because we suspected that there would be carry-over effects from the intervention that may create some additional benefit compared with the pre-intervention controls.

\section{Economic analysis}

We compared the hospital lengths of stay and costs for the three time periods. We specified a two-stage recursive model which exploits the unidirectional causal pathway among the endogenous outcome variables (i.e. length of stay and cost) such that, for a given set of exogenous variables, the endogenous variables can be identified sequentially [35]. The exogenous variables that potentially influenced the length of stay and cost include: patient individual characteristics (age, gender and place of residence), the properties of the presentation (mode of arrival, major diagnostic category and mode of discharge) and whether or not the patient arrived during working hours. Since the recursive model does not allow variables that are linked in a causal chain to have correlated with the error terms, it can control for unobserved heterogeneity and endogeneity within the data $[35,36]$.

\section{Results}

A total of 44,983 records were retrieved (pre-GEDI $n=$ 9066; interim GEDI $n=25,675$; full-GEDI $n=10,242$ ). The patients' mean age was 81 years $(\mathrm{sd}=7)$ and $51 \%$ were female. Most presentations were on weekdays (with peaks on Mondays and Fridays) and between 0800 and $1900 \mathrm{~h}$. There were no important differences between the three time periods for these variables (See Table 1).

There was a small difference in the major diagnostic categories attributed to the three groups with the full intervention sample having a smaller proportion of cardiac presentations $(p<0.001)$, the Interim group having fewer presentations for respiratory conditions $(\mathrm{p}<0.001)$ and the pre-intervention group having fewer presentations for trauma $(\mathrm{p}<0.001)$. More patients arrived by ambulance in the pre-intervention and interim periods than during the full intervention $(\mathrm{p}<0.001)$. Also there were between group differences in ATS category with the full intervention group having a larger proportion in ATS category 2 (urgent, see within $10 \mathrm{~min}$ ) compared to the other time periods $(p<0.001)$. The differences between the three groups noted above were relatively small, and we attempted to minimise the impact of these differences by adjusting for the above variables in our survival models.

\section{Outcomes}

\section{Primary outcome}

The total follow-up time was over 246,000 days. Patients who presented during the GEDI intervention periods, during the GEDI working hours, were more likely to be discharged (See Fig. 1). After $24 \mathrm{~h}$, the discharge probability for the two intervention groups was close to 0.50 , whereas the three control groups were closer to 0.35 (See Fig. 1). The adjusted hazard ratios, for discharge of patients presenting in the GEDI intervention periods, ranged from 1.19 (full intervention during GEDI working hours, $95 \%$ CI $1.13,1.24$ ) to 1.31 (interim intervention outside GEDI working hours, 95\% CI 1.23, 1.39). As expected, many of the patient characteristics influenced discharge, with older patients and those arriving by ambulance less likely to be discharged. There was also a slight trend over time for increased discharge (See Table 2).

There was a reduction in length of hospital stay, for admitted patients, of approximately 1 day between the pre-GEDI time period and the two GEDI intervention periods (Table 3 ). 
Table 1 Demographic and clinical characteristics of the sample

\begin{tabular}{llll}
\hline Characteristic & Pre-implementation Group $\mathrm{n}=9066$ & Interim-implementation Group $n=25,675$ & Full-implementation Group $\mathrm{n}=10,242$ \\
\hline Age - mean $(\mathrm{sd})$ & $81(7)$ & $81(7)$ & $81(7)$ \\
Male gender $-\mathrm{n}(\%)$ & $4349(48)$ & $12,543(49)$ & $5073(50)$ \\
Presenting conditions $-\mathrm{n}(\%)$ & & $2439(24)$ \\
Cardiac & $2441(27)$ & $6586(26)$ & $1860(18)$ \\
Trauma & $1557(17)$ & $4674(18)$ & $914(9)$ \\
Gastrointestinal & $828(9)$ & $2259(9)$ & $883(9)$ \\
Respiratory & $805(9)$ & $2102(8)$ & $865(8)$ \\
Neurological & $735(8)$ & $2221(9)$ & $3273(32)$ \\
Other (20 codes) & $2696(30)$ & $7830(30)$ & $8(<0.1)$ \\
Missing & $4(<0.1)$ & $3(<0.1)$ & $7746(76)$ \\
Arrived by ambulance- $\mathrm{n}(\%)$ & $7196(79)$ & $19,830(77)$ & $146(1)$ \\
Australian Triage Score Category $-\mathrm{n}(\%)$ & & $2554(25)$ \\
Resuscitation & $139(2)$ & $406(2)$ & $4754(46)$ \\
Emergent & $2521(28)$ & $6968(27)$ & $2581(25)$ \\
Urgent & $4219(47)$ & $12,040(47)$ & $207(2)$ \\
Less urgent & $2043(23)$ & $5776(23)$ & $485(2)$ \\
Non-urgent & $144(2)$ & & \\
\hline
\end{tabular}

N.B. Some column percentages do not add to $100 \%$ because of rounding

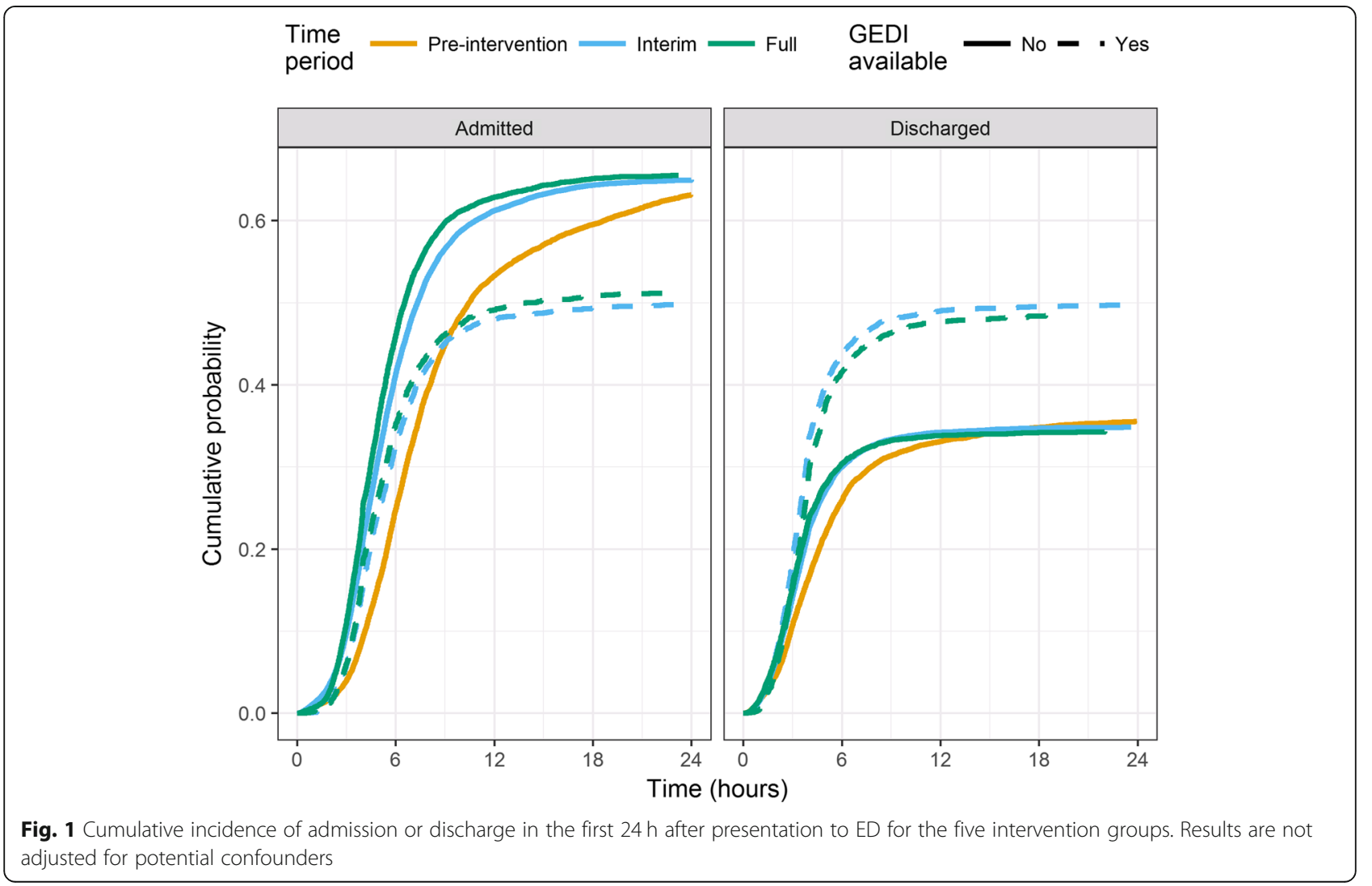


Table 2 Hazard ratios and 95\% confidence intervals for discharge using a Cox survival model with 5 GEDI groups

\begin{tabular}{lll}
\hline Predictor & Hazard Ratio & $95 \% \mathrm{Cl}$ \\
\hline Time (per 100 days increase) & 1.02 & $1.02,1.03$ \\
Age (per 10 year increase) & 0.95 & $0.94,0.97$ \\
Male sex & 1.01 & $0.99,1.04$ \\
Arrival by ambulance & 0.72 & $0.70,0.75$ \\
Australian Triage Score 1 and 2 & 0.94 & $0.90,0.97$ \\
Australian Triage Score 3 & 0.82 & $0.80,0.85$ \\
Presented from RACF & 0.92 & $0.89,0.95$ \\
MDC Diagnosis = Cardiac & 0.95 & $0.92,0.97$ \\
MDC Diagnosis = Trauma & 1.22 & $1.18,1.25$ \\
GEDI group (primary outcome): & & $1.23,1.39$ \\
Interim outside GEDI hours & 1.31 & $1.11,1.29$ \\
Interim during GEDI hours & 1.20 & $1.41,1.53$ \\
Full outside GEDI hours & 1.47 & $1.13,1.24$ \\
Full during GEDI hours & 1.19 &
\end{tabular}

NB Reference for GEDI is pre-intervention. Reference for ATS is 4\&5. Reference for diagnosis is all other matched diagnostic codes Legend: MDC Major diagnostic category

\section{Secondary outcomes}

The length of stay in the ED was shorter for all the GEDI intervention groups compared with pre-intervention (see Table 4). By comparison there was only the likelihood of a shorter hospital length of stay in the interim intervention period during GEDI hours, when compared to pre-GEDI.

There was no clear difference in the risk of death for any of the GEDI periods when compared with pre-GEDI (see Table 4). We note the statistical power for this comparison is relatively low because deaths were rare. The likelihood of a shorter ED or hospital length of stay increased $2 \%$ for every 100 days of the trial.

Re-presentation for the same or any cause based on MDC was not altered by the intervention. The likelihood of re-presentation for other causes was increased if presenting in the interim GEDI period during working hours (see Table 4).

For the economic evaluation, we estimated a number of recursive model specifications to check for robustness and to test the sensitivity of the coefficient estimates. This analysis revealed that when compared to the

Table 3 Comparison of Hospital length of stay (days) by GEDI intervention period using mean differences, 95\% confidence interval for the mean difference and $p$-value from an unpaired ttest

\begin{tabular}{llll}
\hline Comparison & Mean difference & $95 \%$ Cls & P-value \\
\hline Pre vs Interim & -0.96 & $-1.02,-0.90$ & $<0.001$ \\
Pre vs Full & -1.05 & $-1.12,-0.98$ & $<0.001$ \\
Interim vs Full & -0.09 & $-0.13,-0.04$ & $<0.001$ \\
\hline
\end{tabular}

pre-GEDI period, costs per hospital admission in both the full-GEDI and interim-GEDI period were lower (full-GEDI period $=-\$ 1469$ [95\% CI, \$1105, \$1834] and interim-GEDI period $=-\$ 1018$ [95\% CI, \$709, \$1326].

\section{Discussion \\ Principal findings}

In this pragmatic trial we aimed to control for a range of factors that previous studies have shown influence outcomes for older adults who present to the ED. The results indicate that the GEDI model increases the likelihood of discharge, decreases ED and, to some extent, hospital length of stay and costs, with no effect on same cause re-presentation or mortality. Older patients who presented in the months when the GEDI model was in place, and were subsequently admitted to hospital, spent 1 day less in hospital per admission, compared with patients presenting when no GEDI model was implemented and this resulted in cost saving. Other factors also influenced these outcomes with general changes in service provision over time increasing the likelihood of discharge directly from ED.

\section{Strengths and limitations of the study}

This study capitalised on an opportunity to test the effect of a new model of service delivery over the course of its development and once the final version had been determined. Often new models of service delivery are not sustained [27]. Similarly, the final model tested in this intervention did not perform as well as the interim model (possibly representing the enthusiastic efforts of the innovative clinicians that began the change process) but it still outperformed the model employed prior to the commencement of the intervention.

While this pragmatic study used a pre-post design rather than a randomised controlled design to test the effect of the GEDI intervention, design features were incorporated to provide greater generalisability of the results. Our analysis included a variable to adjust for other changes that may have been occurring, in the study ED, over time (for example, maturation of the whole team or other interventions that were introduced to decrease ED length of stay). We also used the survival analysis taking into account major factors identified in the literature that affect patient outcomes for this cohort.

Functional decline and quality of life are important outcomes for this population. However, these variables are not routinely collected in our hospital and hence could not be examined here because of our retrospective study design.

\section{Comparison with other studies}

There are a range of other models of care which aim to improve outcomes for older adults presenting to the ED. 
Table 4 Secondary outcomes for GEDI Intervention

\begin{tabular}{|c|c|c|c|c|}
\hline OUTCOME & $\begin{array}{l}\text { Interim during GEDI hours } \\
\text { Ratio }(95 \% \mathrm{Cl})\end{array}$ & $\begin{array}{l}\text { Interim outside GEDI hours } \\
\text { Ratio }(95 \% \mathrm{CI})\end{array}$ & $\begin{array}{l}\text { Full during GEDI hours } \\
\text { Ratio }(95 \% \mathrm{Cl})\end{array}$ & $\begin{array}{l}\text { Full outside GEDI hours } \\
\text { Ratio }(95 \% \mathrm{CI})\end{array}$ \\
\hline \multirow[t]{2}{*}{ aShorter ED LoS } & 1.40 & 1.48 & 1.28 & 1.42 \\
\hline & $(1.32,1.48)$ & $(1.42,1.54)$ & $(1.19,1.38)$ & $(1.33,1.52)$ \\
\hline \multirow[t]{2}{*}{ 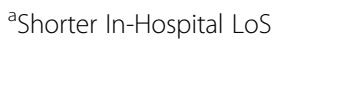 } & 1.15 & 1.04 & 1.00 & 0.98 \\
\hline & $(1.07,1.23)$ & $(0.99,1.09)$ & $(0.91,1.11)$ & $(0.90,1.06)$ \\
\hline \multirow[t]{2}{*}{${ }^{\mathrm{b}}$ Risk of Death } & 0.32 & 0.52 & 1.01 & 0.77 \\
\hline & $(0.08,1.08)$ & $(0.23,1.14)$ & $(0.23,4.43)$ & $(0.18,3.22)$ \\
\hline \multirow{2}{*}{$\begin{array}{l}\text { aLess same cause ED re- } \\
\text { presentation within } 28 \text { days }\end{array}$} & 1.13 & 1.04 & 1.21 & 1.19 \\
\hline & $(0.89,1.42)$ & $(0.89,1.22)$ & $(0.88,1.66)$ & $(0.89,1.58)$ \\
\hline \multirow{2}{*}{$\begin{array}{l}\text { aLess any cause ED re-present } \\
\text { within } 28 \text { days }\end{array}$} & 1.18 & 1.06 & 1.21 & 1.10 \\
\hline & $(1.02,1.37)$ & $(0.96,1.18)$ & $(0.99,1.49)$ & $(0.91,1.32)$ \\
\hline
\end{tabular}

${ }^{a}$ hazard ratio, ${ }^{b}$ prevalence ratio, NB Reference for GEDI is pre-intervention

Some are outreach models or Hospital in the Nursing Home models in which ED or hospital clinicians travel to RACFs aiming to prevent transfer [15, 37-40]. These outreach models, unlike the GEDI model, may de-skill RACF staff and result in general medical practitioner disengagement. They may also be very expensive, although it is difficult to understand cost as few robust cost analyses have been undertaken [1]. Additionally, these models only work for the small proportion of older adults living in RACFs.

Other models focus on enhancing the care within the ED. The senior work up assessment and treatment (SWAT) model [41], the Triage and Rapid Elderly Assessment Team (TREAT) model [12], the Aged Care Services Emergency Team (ASET) Program [42], the GEDI WISE transitional care nurses [43] and a range of other models that combine some level of increased geriatric assessment and liaison with community services [44] have been described. Of these models only the first three report rigorous evaluative research outcomes. Unlike our GEDI model the SWAT [41] model, of increased senior medical officer involvement, did not improve overall ED length of stay but did improve length of stay on high volume days and for discharged patients. The TREAT [12] model which involved a highly skilled specialist team of consultant geriatricians and physicians, nurse practitioners and allied health staff, did reduce hospital admission but as there was no economic evaluation it is not possible to compare the costs of this service with the GEDI model. The GEDI WISE [43] transitional care model is the model most similar to GEDI and also resulted in decreased admissions. However, there is no specific physician champion in the GEDI WISE model and the increase in re-presentation seen in their model, but not seen in our GEDI evaluation, may be because of the important role played by the senior medical officer. The physician champion role, in this nurse-led model, is unique to GEDI and ensures there is multi-disciplinary involvement and that the care of older adults is seen as being as important as the care of other cohorts within the ED. In addition, the provision of post ED support to prevent re-presentations in the community dwelling cohort is dependent upon a supportive community-based health network. The ED physician champion plays a role in ensuring collaboration between the ED setting and community health services to provide continuity of client care.

\section{Meaning of the study}

Focusing on improving the care of older adults in the ED and on strategies to prevent inappropriate ED presentation by older adults is of increasing importance in light of population ageing. Sinha and colleagues [17] identified the eight components of ED-based models of care for non-institutionalised older adults. The GEDI model was developed to include all of these components. A key finding of Sinha et al. [17] was that, "collaborative working practices are critical in model implementation and rely on the interpersonal skill sets of the clinicians delivering those initiatives and their ability to earn the trust and respect of their colleagues within and beyond the ED" (p. 680). Our findings suggest that it is possible to have advanced practice nurses, rather than nurse practitioners, in the GEDI model provided there is a strong physician-champion who supports, advises and works collaboratively with the senior nurse. This provides a relatively inexpensive yet effective model of care that reduces length of stay and increases discharge of older adults from the ED, when appropriate.

\section{Conclusions}

There is a need for senior managers and policy makers to reconsider the models of care employed in ED service delivery for vulnerable groups. It is well accepted that 
emergency care for paediatric patients requires specialized resources including equipment, drugs, trained personnel, and facilities [45]. Yet for other vulnerable groups the provision of specialist care within the ED or increased training in a specialty for ED clinicians is not recognised. The results of this study suggest that having teams of emergency clinicians (doctors and nurses) with previous training and experience in geriatrics and community care, who are focused on streamlining the care of frail older adults in the ED, improves outcomes and cuts costs. Furthermore, these benefits can be sustained over time and while they may slightly decrease when the innovative team that effects change have moved to more senior roles, overall improvement remains. Finding solutions that maximise the outcomes for older people living in RACFs, who develop an acute illness, will involve greater inter-sectoral collaboration and require further research.

\section{Abbreviations}

CEDRiC project: Care coordination through Emergency Department, Residential Aged Care and Primary Health Collaboration project; ED: Emergency Department; GEDI: Geriatric Emergency Department Intervention; HREC: Human Research Ethics Committee; PHN: Primary health network; RACF: Residential aged care facility

\section{Acknowledgements \\ Expert advisory committee members contributing to development of the intervention - Dr. Ruth Devin, Geriatrician; Dr. Zoltan Bourne, General Practitioner; Sylvia Hood Assistant Manager, Health Information Services SCHHS; Barry McCarthy, Nursing Director, SCHHS; Matt Sierp, Chief Operating Officer, Sundale Ltd.; Pattie Hudson, Chief Executive Officer, Central Queensland, Wide Bay and Sunshine Coast PHN; Mr. Graham Chapman, Aged Care Consumer Representative; Mrs. Joan Chapman, Aged Care Consumer Representative and Volunteer for marketing and promotion of service; Morag Oakley, Clinical Services Director, Sundale Ltd.; Nicolette Bannink, Research Assistant, USC; and study participants.}

\section{Funding}

This research was supported by a grant from the Australian Government Aged Care Service Improvement and Healthy Ageing Grant Scheme and a grant from the Wishlist Foundation. Neither the Australian Government nor the Wishlist Foundation had a role in any aspect of the study or decisions about interpretation of results or publication. All authors had full access to all of the data (including statistical reports and tables) in the study and can take responsibility for the integrity of the data and the accuracy of the data analysis.

\section{Availability of data and materials}

To guarantee the confidentiality of anonymised patient data and health information only the authors have had access to the de-identified data during the study. The linked data were provided by the hospital and health service under a research agreement that does not permit sharing.

\section{Authors' contributions}

The development of the intervention was led by EM and AT with input from MW, JC, AG and members of the external advisory committee. The research study was planned by MW and EM with input from all other authors and input from members of the advisory committee. The analysis of data was planned and executed by $A B$ in consultation with MW, AC and EM. The health economics component was undertaken by $\mathrm{K}-\mathrm{HN}$ in consultation with $\mathrm{MW}, \mathrm{AC}$ and EM. The project was managed by $\mathrm{AC}$ assisted by $\mathrm{MB}$ and $\mathrm{CJ}$ with input from all authors and the external advisory committee. MW led the interpretation of the data and the drafting of the manuscript with help from all other authors. All authors contributed to revisions of the manuscript. All authors read and approved the final manuscript.

\section{Ethics approval and consent to participate}

This study received ethics approval and approval for a waiver of consent (as the data used were de-identified retrospectively accessed clinical and financial records) from The Prince Charles' Hospital Human Research Ethics Committee (HREC), University of Sunshine Coast HREC with associated Public Health Act and site specific approvals for the relevant Hospital and Health Service as per the following list. HREC/14/QPCH/220, HREC/15/QPCH/290, A/15/718, SSA/15/ QNB/40 and SSA/16/QNB/17. Agreements were in place between all partner organisations.

\section{Consent for publication}

N/A. No individual data are presented.

\section{Competing interests}

All authors have completed the ICMJE uniform disclosure form at www.icmje.org/coi_disclosure.pdf and declare: that this study was supported by competitive grants from the Australian Government (Department of Social Services and Department of Health) and the Wishlist Foundation; that this study was supported financially by the Sunshine Coast Medicare Local and received in kind support from the Sunshine Coast Hospital and Health Service and the University of the Sunshine Coast; no financial relationships with any organisations that might have an interest in the submitted work in the previous 3 years; no other relationships or activities that could appear to have influenced the submitted work.

\section{Publisher's Note}

Springer Nature remains neutral with regard to jurisdictional claims in published maps and institutional affiliations.

\section{Author details \\ ${ }^{1}$ School of Nursing, Midwifery and Paramedicine, University of Sunshine Coast, 90 Sippy Downs Drive, Sippy Downs, QLD 4556, Australia. ${ }^{2}$ Sunshine Coast and Hospital Health Service, Emergency Services, Birtinya, QLD, Australia. ${ }^{3}$ Institute of Health and Biomedical Innovation \& School of Public Health and Social Work, Queensland University of Technology, Kelvin Grove, QLD 4059, Australia. ${ }^{4}$ Center for Health Service Research, Faculty of Medicine, University of Queensland, Herston, QLD 4006, Australia. ${ }^{5}$ Program Management Office, Central Queensland, Wide Bay, Sunshine Coast PHN, Ground Floor, Mayfield House, 29 The Esplanade, Maroochydore, QLD 4558, Australia. ${ }^{6}$ Menzies Health Institute Queensland, Griffith University, Gold Coast Campus, Griffith, QLD 4222, Australia. ${ }^{7}$ Department of Emergency Medicine, Gold Coast Health, Southport, QLD, Australia.}

Received: 10 July 2018 Accepted: 22 November 2018

Published online: 03 December 2018

\section{References}

1. Arendts $G$, Howard $K$. The interface between residential aged care and the emergency department: a systematic review. Age Ageing. 2010;39(3):306-12.

2. Australian Institute of Health and Welfare. Residential aged care in Australia 2010-11: a statistical overview Canberra: AlHW; 2012.

3. Australian Institute of Health and Welfare. Aged care packages in the community 2010-11: a statistical overview. Canberra: AlHW; 2012.

4. Hwang U, Shah MN, Han JH, Carpenter CR, Siu AL, Adams JG. Transforming emergency care for older adults. Health Aff. 2013;32(12):2116-21.

5. Aminzadeh F, Dalziel WB. Older adults in the emergency department: a systematic review of patterns of use, adverse outcomes, and effectiveness of interventions. Ann Emerg Med. 2002;39(3):238-47.

6. Covinsky KE, Palmer RM, Fortinsky RH, Counsell SR, Stewart AL, Kresevic D, et al. Loss of independence in activities of daily living in older adults hospitalized with medical illnesses: increased vulnerability with age. J Am Geriatr Soc. 2003;51(4):451-8.

7. Creditor MC. Hazards of hospitalization of the elderly. Ann Intern Med. 1993;118(3):219-23.

8. Inouye SK, Bogardus ST Jr, Charpentier PA, Leo-Summers L, Acampora D, Holford TR, et al. A multicomponent intervention to prevent delirium in hospitalized older patients. N Engl J Med. 1999;340(9):669-76.

9. Crilly J, Chaboyer W, Wallis M, Thalib L, Green D. Predictive outcomes for older people who present to the emergency department. Australas Emerg Nurs J. 2008;11(4):178-83. 
10. Mudge AM, Denaro CP, O'Rourke P. Improving hospital outcomes in patients admitted from residential aged care: results from a controlled trial. Age Ageing. 2012;41(5):670-3.

11. Silvester KM, Mohammed MA, Harriman P, Girolami A, Downes TW. Timely care for frail older people referred to hospital improves efficiency and reduces mortality without the need for extra resources. Age Ageing. 2014;43(4):472-7.

12. Wright PN, Tan G, lliffe $\mathrm{S}$, Lee $\mathrm{D}$. The impact of a new emergency admission avoidance system for older people on length of stay and same-day discharges. Age Ageing. 2014;43(1):116-21.

13. Arendts $G$, Reibel T, Codde J, Frankel J. Can transfers from residential aged care facilities to the emergency department be avoided through improved primary care services? Data from qualitative interviews. Australas J Ageing. 2010;29(2):61-5.

14. Codde J, Arendts G, Frankel J, Ivey M, Reibel T, Bowen S, et al. Transfers from residential aged care facilities to the emergency department are reduced through improved primary care services: an intervention study. Australas J Ageing. 2010;29(4):150-4.

15. Crilly J, Chaboyer W, Wallis M, Thalib L, Polit D. An outcomes evaluation of an Australian Hospital in the Nursing Home admission avoidance programme. J Clin Nurs. 2011;20(7-8):1178-87.

16. Hastings SN. Heflin MT. a systematic review of interventions to improve outcomes for elders discharged from the emergency department. Acad Emerg Med Off J Soc Acad Emerg Med. 2005;12(10):978-86.

17. Sinha SK, Bessman ES, Flomenbaum N, Leff B. A systematic review and qualitative analysis to inform the development of a new emergency department-based geriatric case management model. Ann Emerg Med. 2011;57(6):672-82

18. Conroy SP, Ansari K, Williams M, Laithwaite E, Teasdale B, Dawson J, et al. A controlled evaluation of comprehensive geriatric assessment in the emergency department: the 'Emergency frailty unit. Age Ageing. 2014:43(1):109-14

19. Shih SL, Gerrard P, Goldstein R, Mix J, Ryan CM, Niewczyk P, et al. Functional status outperforms comorbidities in predicting acute care readmissions in medically complex patients. J Gen Intern Med. 2015;30(11):1688-95.

20. American College of Emergency Physicians. Geriatric emergency department guidelines. Ann Emerg Med. 2014;63(5):e7-25.

21. Jennings N, Gardner G. O'Reilly G. a protocol for a pragmatic randomized controlled trial evaluating outcomes of emergency nurse practitioner service. J Adv Nurs. 2012;68(1):2140-8.

22. Crilly J, Chaboyer W, Wallis M. Continuity of care for acutely unwell older adults from nursing homes. Scand J Caring Sci. 2006;20(2):122-34.

23. Goldstein J, Travers A, Hubbard R, Moorhouse P, Andrew MK, Rockwood K Assessment of older adults by emergency medical services: methodology and feasibility of a care partner comprehensive geriatric assessment (CP-CGA) CJEM. 2014;16(0):1-14.

24. Donabedian A. An introduction to quality Assurance in Health Care. Oxford: Oxford University Press; 2003.

25. Marsden E, Taylor A, Wallis M, Craswell A, Broadbent M, Barnett A, et al. A structure, process and outcome evaluation of the geriatric emergency department intervention model of care: a study protocol. BMC Geriatr. 2017;17(1):76.

26. Sanson-Fisher RW, Bonevski B, Green LW, D'Este C. Limitations of the randomized controlled trial in evaluating population-based health interventions. Am J Prev Med. 2007;33(2):155-61.

27. NHS Institute. Sustainability and its relationship with spread and adoption. Coventry: NHS Institute for Innovation and Improvement; 2007.

28. Australasian College for Emergency Medicine. Guidelines for the Implementation of the Australasian Triage Scale. Melbourne; 2013. p. 2013 Report No.: G24

29. Australian Commission on Safety and Quality in Health Care. Adult Deterioration Detection System (ADDS) chart 2010. https://www. safetyandquality.gov.au/wp-content/uploads/2012/01/35981ChartDevelopment.pdf. Accessed 9 June 2018.

30. Craswell A, Marsden E, Taylor A, Wallis M. Emergency department presentation of frail older people and interventions for management: geriatric emergency department intervention. Safety in Health. 2016;2(14):6.

31. Marsden E, Taylor A, Wallis M, Craswell A, Bannink N, Broadbent M, et al. Geriatric emergency department intervention toolkit. 2017. http://www. cedric.org.au/Toolkit.php. Accessed 9 June 2018.
32. Wolkewitz M, Cooper BS, Bonten MJ, Barnett AG, Schumacher M. Interpreting and comparing risks in the presence of competing events. BMJ (Clinical research ed). 2014;349:95060.

33. Shadish WR, Cook TD, Campbell DT. Experimental and quasi-experimental designs for generalized causal inference. Boston: Houghton Mifflin; 2002. p. 623.

34. Barnett AG, Dobson AJ. Analysing seasonal health data. Berlin: Springer; 2010

35. Cortina LM, Models R. In: Everitt B, Howell D, editors. Encyclopedia of statistics in behavioral science. Hoboken, N.J: John Wiley \& Sons; 2005. p. 1722-3.

36. Rowell D, Nguyen $\mathrm{K}-\mathrm{H}$, Baade $\mathrm{P}$, Janda M. Evaluation of a skin self-examination Programme: a four-stage recursive model. Asian Pac J Cancer Prev. 2017;18(4):1063-7.

37. Burkett E, Scott I. CARE-PACT: a new paradigm of care for acutely unwell residents in aged care facilities. Aust Fam Physician. 2015;44(4):204-9.

38. Conway J, Dilworth S, Hullick C, Hewitt J, Turner C, Higgins I. A multiorganisation aged care emergency service for acute care management of older residents in aged care facilities. Aust Health Rev. 2015;39(5):514-6.

39. Conway J, Higgins I, Hullick C, Hewitt J, Dilworth S. Nurse-led ED support for residential aged care facility staff: an evaluation study. Int Emerg Nurs. 2015:23(2):190-6.

40. Fan L, Xiang-Yu H, Zhao J, Sun J, Dingle K, Purtill R, et al. Hospital in the nursing home program reduces emergency department presentations and hospital admissions from residential aged care facilities in Queensland, Australia: a quasi-experimental study. BMC Health Serv Res. 2016;16:46.

41. Davis RA, Dinh MM, Bein KJ, Veillard AS, Green TC. Senior work-up assessment and treatment team in an emergency department: a randomised control trial. Emergency medicine Australasia: EMA. 2014;26(4):343-9.

42. Shanley C, Sutherland S, Tumeth R, Stott K, Whitmore E. Caring for the older person in the emergency department: the ASET program and the role of the ASET clinical nurse consultant in South Western Sydney, Australia. J Emerg Nurs. 2009:35(2):129-33.

43. Hwang U, Dresden SM, Rosenberg MS, Garrido MM, Loo G, Sze J, et al. Geriatric emergency department innovations: transitional care nurses and hospital use. J Am Geriatr Soc. 2018;66(3):459-66.

44. Lowthian J, McGinnes R, Brand C, Barker A, Cameron P. Discharging older patients from the emergency department effectively: a systematic review and meta-analysis. Age Ageing. 2015:44(5):761-70.

45. American Academy of Pediatrics Committee on Pediatric Emergency Medicine, et al. Joint policy statement-guidelines for Care of Children in the emergency department. Pediatrics. 2009;124(4):1233-43.

\section{Ready to submit your research? Choose BMC and benefit from:}

- fast, convenient online submission

- thorough peer review by experienced researchers in your field

- rapid publication on acceptance

- support for research data, including large and complex data types

- gold Open Access which fosters wider collaboration and increased citations

- maximum visibility for your research: over $100 \mathrm{M}$ website views per year

At $\mathrm{BMC}$, research is always in progress.

Learn more biomedcentral.com/submissions 